\title{
DA CANZONI (E POESIE) A CANZONI. ADATTAMENTI METRICI NELLE TRADUZIONI DI FABRIZIO DE ANDRÉ
}

\author{
DE CANCIONES (Y POEMAS) A CANCIONES. \\ LAS TRADUCCIONES DE FABRIZIO DE ANDRÉ
}

FROM SONGS (AND POEMS) TO SONGS. FABRIZIO DE ANDRÉ'S TRANSLATIONS

\author{
Gianluca VALENTI \\ Université Catholique de Louvain (UCL) \\ gianluca_valenti@yahoo.it
}

Resumen: El presente trabajo reflexiona sobre las modalidades de traducción métrica de poemas a canciones, y de canciones, inglesas y francesas, asimismo a canciones en italiano. El análisis demuestra que los esquemas métricos y los patrones melódicos permanecen totalmente inalterados cuando el traductor traduce versos cantados, pero no cuando lo traducido son poemas. Además, el estudio demuestra que el idioma del texto original no afecta a las elecciones métricas del traductor. Se ha analizado el corpus lírico de Fabrizio De André. 
Abstract: Aim of this article is to investigate the modalities of metrical translation from poems and songs to songs. To this aim I chose a subset of Fabrizio De André's songs which are translations both from poems and from French and English songs. In particular, I show that the metrical grid and the melodic pattern are fully respected during the transposition from sung verse to sung verse; contrarily, the adaptation of poems into sung verse shows a high level of metrical freedom in the translated texts. While the language of the original text seems to barely influence the metrical grid of De André's songs, the presence of the original melody is a major constraint in the metrical choices of the translator.

Palabras clave: Traducción. De André. Brassens. Cohen. Masters.

Key Words: Translation. De André. Brassens. Cohen. Masters.

\section{LA TRADUZIONE METRICA}

La traduzione di un testo da un sistema linguistico a un altro ha sempre creato notevoli problemi a chi con essa si è confrontato, soprattutto nel caso in cui - oltre agli aspetti semantici, sintattici, fonici...- il traduttore si sia trovato costretto a considerare anche vincoli di tipo metrico. L'analisi delle modalità con cui tali traduzioni vengono approntate è argomento attualmente di estremo interesse, e ancora lungi dall'essere risolto in via definitiva ${ }^{1}$.

L'oggetto di studio del presente articolo sono le implicazioni metriche della traduzione di testi di vario tipo, che hanno in comune l'essere vincolati alle regole della metrica (sono dunque esclusi dall'analisi i testi in prosa), in brani destinati ad essere musicati e cantati. Prescindendo da altre variabili, non ultime quelle di tipo cognitivo - isolate e studiate, in parte, in altri contesti (Valenti, 2011) - , la domanda a cui si risponderà è la seguente: il modo in cui opera il traduttore è soggetto a variazioni significative in base sia al linguaggio, sia al genere letterario del testo di partenza? Nello specifico, si analizzeranno le traduzioni (di poesie e canzoni, tanto francesi quanto inglesi) effettuate dal cantautore genovese Fabrizio De André. La scelta di componimenti appartenenti a un unico artista permetterà di lavorare su un corpus di testi omogeneo, in modo da ricavare dei dati fra loro commensurabili.

${ }^{1}$ Sul problema della traduzione nel verso cantato sono recentemente intervenuti, al Convegno internazionale «Metrics, Music and Mind. Linguistic, Metrical and Cognitive Implications in Sung Verse» (Roma, 23-25 Febbraio 2012), D’Andrea, Franzon, Greenall, Kaindl e Smith-Sivertsen. 
Si possono brevemente anticipare alcuni dei risultati che verranno in seguito argomentati in forma analitica.

L'esito di maggiore interesse della presente ricerca risiede nell'incontestabile riscontro di una rigorosa fedeltà alla struttura metrica del testo di partenza qualora vengano tradotti dei versi cantati, e - di contro - di un'assoluta libertà metrica qualora invece vengano tradotti dei testi poetici, privi dunque di qualsiasi legame con la musica. Nonostante a prima vista tale risultato possa sembrare controintuitivo - essendo la poesia il genere per eccellenza coinvolto con la metrica-, si può avanzare una possibile spiegazione a tale fenomeno. Difatti, mentre il cantautore che traduce una poesia può trasgredire le norme metriche del testo di partenza nel nome di una maggiore aderenza ad altri vincoli (come ad esempio quello della fedeltà semantica), il traduttore di canzoni è invece obbligato a rispettare devotamente lo schema del testo originale, poiché un'elevata quantità di variazioni metriche comporterebbe di conseguenza uno stravolgimento della melodia su cui il proprio testo è modulato.

In secondo luogo, sembra invece che il supporto linguistico del brano originale non incida in maniera significativa sugli esiti della traduzione. È ovvio che più la lingua di partenza si allontana dalla lingua del traduttore e più la traduzione risulterà problematica; tuttavia, analizzando brani di De André che traducono canzoni scritte in una lingua romanza (francese) e canzoni scritte in una lingua germanica (inglese), non si sono riscontrate variazioni tali da essere definitivamente imputabili a differenze di ordine linguistico.

\section{DA CANZONI (FRANCESI) A CANZONI}

In questa sezione iniziale si esamineranno le traduzioni, dal francese all'italiano, di testi cantati; a conclusione dell'analisi si riscontrerà una forte somiglianza di metodo nella tecnica di traduzione utilizzata per tutti i brani presi in esame.

A supporto della bontà di tale risultato si può aggiungere che, se i brani italiani fossero stati incisi tutti nel medesimo periodo, sarebbe stato legittimo obiettare che le similarità delle modalità di traduzione potessero essere dovute a contingenze di vario tipo, come ad esempio una particolare forma di espressione artistica che il cantautore sperimentò in un limitato arco temporale. Di contro, il fatto che i tre brani siano apparsi a distanza di diversi anni l'uno dall'altro mostra come il metodo messo in atto fosse l'unico - almeno 
per ciò che concerne la traduzione di testi cantati in lingua francese - che De André riteneva funzionale per i suoi obiettivi.

\subsection{Attenti al gorilla!}

«Il gorilla» (1968) è una delle primissime traduzioni di canzoni francesi effettuata da Fabrizio De André.

\section{Le gorille (Brassens)}

C'est à travers de larges grilles

Que les femelles du canton

Contemplaient un puissant gorille

Sans souci du qu'en-dira-t-on

Avec impudeur ces commères

Lorgnaient même un endroit précis

Que rigoureusement ma mère

M'a défendu de nommer ici

Gare au gorille

Tout à coup la prison bien close

Où vivait le bel animal

S'ouvre on n'sait pourquoi je suppose

Qu'on avait du la fermer mal

Le singe en sortant de sa cage

Dit "c'est aujourd'hui que je le perds"

Il parlait de son pucelage

Vous aviez deviné j'espère

Gare au gorille

L'patron de la ménagerie

Criait éperdu "nom de nom

C'est assommant car le gorille

N'a jamais connu de guenon"

\section{Il gorilla (De André)}

Sulla piazza d'una città

La gente guardava con ammirazione

Un gorilla portato là

Dagli zingari d'un baraccone

Con poco senso del pudore

Le comari di quel rione

Contemplavano l'animale

Non dico come non dico dove

Attenti al gorilla

D’improvviso la grossa gabbia

Dove viveva l'animale

S'aprì di schianto non so perché

Forse l'avevano chiusa male

La bestia uscendo fuori di là

Disse "quest'oggi me la levo"

Parlava della verginità

Di cui ancora viveva schiavo

Attenti al gorilla

Il padrone si mise a urlare

"Il mio gorilla fate attenzione

Non ha veduto mai una scimmia

Potrebbe fare confusione" 
Dès que la féminine engeance Sut que le singe était puceau Au lieu de profiter de la chance Elle fit feu des deux fuseaux

Gare au gorille

Celles là même qui naguère

Le couvaient d'un oil décidé

Fuirent prouvant qu'elles n'avaient guère

De la suite dans les idées

D'autant plus vaine était leur crainte Que le gorille est un luron Supérieur à l'homme dans l'étreinte Bien des femmes vous le diront

Gare au gorille

Tout le monde se précipite

Hors d'atteinte du singe en rut

Sauf une vielle décrépite

Et un jeune juge en bois brut

Voyant que toutes se dérobent

Le quadrumane accéléra

Son dandinement vers les robes

De la vieille et du magistrat

Gare au gorille

"Bah" soupirait la centenaire

"Qu'on put encore me désirer

Ce serait extraordinaire

Et pour tout dire inespéré"

Le juge pensait impassible

"Qu'on me prenne pour une guenon

C'est complètement impossible"

La suite lui prouva que non
Tutti i presenti a questo punto

Fuggirono in ogni direzione

Anche le donne dimostrando

La differenza fra idea e azione

Attenti al gorilla

Tutta la gente corre di fretta

Di qua e di là con grande foga

Si attardano solo una vecchietta

E un giovane giudice con la toga

Visto che gli altri avevan squagliato

Il quadrumane accelerò

E sulla vecchia e sul magistrato

Con quattro salti si portò

\section{Attenti al gorilla}

“Bah” sospirò pensando la vecchia

"Ch'io fossi ancora desiderata

Sarebbe cosa alquanto strana

E più che altro non sperata"

"Che mi si prenda per una scimmia"

Pensava il giudice col fiato corto

"Non è possibile, questo è sicuro"

Il seguito prova che aveva torto

Attenti al gorilla 
Gare au gorille

Supposez qu'un de vous puisse être Comme le singe obligé de

Violer un juge ou une ancêtre

Lequel choisirait-il des deux

Qu'une alternative pareille Un de ces quatres jours m'échoie C'est j'en suis convaincu la vieille Qui sera l'objet de mon choix

Gare au gorille

Mais par malheur si le gorille Aux jeux de l'amour vaut son prix On sait qu'en revanche il ne brille Ni par le goût ni par l'esprit

Lors au lieu d'opter pour la vieille Comme l'aurait fait n'importe qui Il saisit le juge à l'oreille Et l'entraîna dans un maquis

Gare au gorille

La suite serait délectable Malheureusement je ne peux Pas la dire et c'est regrettable Ça nous aurait fait rire un peu

Car le juge au moment suprême Criait "maman" pleurait beaucoup Comme l'homme auquel le jour même Il avait fait trancher le cou

Gare au gorille
Se qualcuno di voi dovesse Costretto con le spalle al muro Violare un giudice od una vecchia Della sua scelta sarei sicuro

Ma si dà il caso che il gorilla Considerato un grandioso fusto Da chi l'ha provato però non brilla Né per lo spirito né per il gusto

Attenti al gorilla

Infatti lui sdegnanta la vecchia Si dirige sul magistrato

Lo acchiappa forte per un'orecchia E lo trascina in mezzo a un prato

Quello che avvenne tra l'erba alta Non posso dirlo per intero Ma lo spettacolo fu avvincente E la suspence ci fu davvero

Attenti al gorilla

Dirò soltanto che sul più bello Dello spiacevole e cupo dramma Piangeva il giudice come un vitello Negli intervalli gridava "mamma"

Gridava "mamma" come quel tale Cui il giorno prima come ad un pollo Con una sentenza un po' originale Aveva fatto tagliare il collo

Attenti al gorilla 
La canzone francese è strutturata su nove strofe, ciascuna composta di otto octosyllabes con una forte pausa melodica al mezzo, seguite da un ritornello di un verso con un lungo melisma sull'ultima sillaba accentata ${ }^{2}$; si noti inoltre l'artificio di disporre rime maschili per i versi pari, e rime femminili per i versi dispari, ottenendo così lo schema: 8'a8b8'a8b8'c8d8'c8d³ . Di seguito si riproduce la griglia dei primi quattro versi del brano di Brassens e della traduzione di De André, in accordo con la proposta di Liberman (1975), Lerdahl, Jackendoff (1983) e anche Hayes, Kaun (1996: 244-249) e Halle, Lerdahl (1993):

\begin{tabular}{|c|c|c|c|c|c|c|c|c|c|c|c|c|}
\hline (A) & I & II & III & IV & $\mathbf{V}$ & VI & VII & VIII & IX & $\mathbf{X}$ & XI & XII \\
\hline & & & & & $\mathrm{X}$ & & & & & & $\mathrm{X}$ & \\
\hline & & $\mathrm{X}$ & & & $\mathrm{x}$ & & & $\mathrm{X}$ & & & $\mathrm{X}$ & \\
\hline & & $\mathrm{X}$ & $\mathrm{X}$ & $\mathrm{X}$ & $\mathrm{X}$ & $X$ & $\mathrm{x}$ & $\mathrm{X}$ & $\mathrm{X}$ & $\mathrm{X}$ & $\mathrm{X}$ & $\mathrm{X}$ \\
\hline 1 & & c'est & à & tra- & vers & & de & lar- & & ges & gri- & lles \\
\hline 2 & & sul- & la & & piaz- & $\mathrm{za}$ & & d'u- & na & cit- & tà & \\
\hline 3 & & que & les & fe- & me- & & lles & $\mathrm{du}$ & can- & & ton & \\
\hline 4 & la & gen- & te & guar- & da- & va & con & am- & mi- & ra- & zio- & ne \\
\hline 5 & & con- & & tem- & plaient & un & pui- & ssant & & go- & ri- & 1le \\
\hline 6 & & un & & go- & ril- & la & por- & ta- & to & & là & \\
\hline 7 & & sans & sou- & ci & $\mathrm{du}$ & & qu'en & di- & ra- & & t-on & \\
\hline 8 & & da- & gli & & zin- & ga- & ri & d'un & ba- & rac- & co- & \\
\hline
\end{tabular}

${ }^{2}$ A partire dal quale è stato anche suggerito che «l'existence d'exemples comme le refrain du Gorille de Brassens suggère qu'il ne faut pas essayer d'imposer une limite supérieure au nombre de notes (ou de mesures) sur lequel une syllabe peut être étirée» (Dell, 1989: 130).

${ }^{3}$ Ma si veda pure la diversa formalizzazione proposta da Hayes, McEachern (1998). 
La traduzione di De André, pur possedendo una strofa in meno dell'originale e pur ostentando una maggiore libertà, rispetto al testo francese, sia nell'alternare novenari a decasillabi, sia nell'applicazione - rispettata solo in parte - del medesimo schema di rime, può considerarsi tuttavia estremamente fedele da un punto di vista metrico-melodico.

Difatti, mantenendo immutata la melodia, il cantautore italiano ha dovuto mantenere altrettanto immutata la struttura metrica, sacrificando a essa, in alcuni punti, il lessico e persino il senso generale della frase. Del tutto secondario, e metricamente ininfluente, il mancato rispetto del numero di sillabe del testo originale perché -com'è particolarmente evidente alle linee 3-4 della griglia - anche qualora le sillabe del brano italiano eccedano la misura originaria, il numero di tempi metrico-ritmici in cui il testo si inserisce è identico a quello dell'originale francese. Già da questo primo esempio si può notare che il legame melodico con la canzone di partenza era uno dei vincoli che De André era piú propenso a rispettare.

Del resto, il confronto fra la prima strofa della canzone di De André e un'ipotetica traduzione letterale di quella di Brassens rende bene l'idea di quanto, da un punto di vista strettamente lessicale, i due testi siano distanti fra loro.

\section{Le gorille (traduzione letterale)}

$\grave{E}$ a traverso larghe sbarre che le femmine del distretto contemplavano un potente gorilla senza preoccupazione di cosa se ne dirà

senza pudore queste comari adocchiavano pure un posto specifico che rigorosamente mia madre mi ha proibito di nominare qui

\section{Il gorilla (De André)}

Sulla piazza d'una città la gente guardava con ammirazione un gorilla portato là dagli zingari d'un baraccone

con poco senso del pudore le comari di quel rione contemplavano l'animale non dico come non dico dove

Il rispetto dello schema di rime, mantenuto nella prima metà della strofa italiana, viene meno nella seconda, dove aumenta però la fedeltà all'originale per ciò che concerne la lunghezza dei singoli versi. In entrambi i casi si nota facilmente come della canzone francese si sia conservato, in maniera molto approssimativa, il significato complessivo della strofa, ma di quasi ogni verso sia passata completamente in secondo piano l'aderenza letterale al testo. 
Dunque, lì dove il vincolo lessicale (e talvolta addirittura semantico) entra in contrasto con il vincolo metrico, sarà quest'ultimo ad avere il sopravvento. La divergenza del brano italiano dall'originale si deve infatti alla mera impossibilità del cantautore di conciliare tali costrizioni, e non certo alla sua volontà di produrre un testo diverso da quello di partenza. Il secondo esempio qui riprodotto (par. 2.2) mostra infatti come, qualora la griglia metrica non precluda la possibilità di una traduzione letterale, sarà proprio quest'ultima soluzione ad essere preferita.

\subsection{L'idea è affascinante}

Alcuni anni dopo la composizione de «Il gorilla» esce l'album «Canzoni» (1974), i cui unici brani inediti sono tutti esito di traduzioni. La tecnica di De André, nel frattempo, si è affinata, e il cantautore riesce ora ad abbinare a una riproposizione pressoché perfetta dello schema metrico-melodico del testo di partenza una resa letterale della traduzione dei singoli vocaboli. Di seguito la prima strofa di entrambi i testi:

\section{Mourir pour des idées (Brassens) Morire per delle idee (De André)}

Mourir pour des idées

l'idée est excellente

moi j'ai failli mourir

de ne l'avoir pas eu

car tous ceux qui l'avaient

multitude accablante

en hurlant à la mort

me sont tombés dessus

ils ont su me convaincre

et ma muse insolente

abjurant ses erreurs

se rallie à leur foi

avec un soupçon de

réserve toutefois

mourrons pour des idées

d'accord mais de mort lente

d'accord mais de mort lente
Morire per delle idee

l'idea è affascinante

Per poco io morivo

senza averla mai avuta

Perchè chi ce l'aveva

una folla di gente

Gridando "viva la morte"

proprio addosso mi è caduta

Mi avevano convinto

e la mia musa insolente

Abiurando i suoi errori

aderi alla loro fede

Dicendomi peraltro

in separata sede

Moriamo per delle idee

Vabbè ma di morte lenta

Vabbè ma di morte lenta 
Nonostante il maggior numero di sillabe necessarie all'italiano per una traduzione letterale di vocaboli francesi, la canzone di De André segue quasi perfettamente il pattern ritmico del brano di partenza ${ }^{4}$, come si può osservare dalle griglie sottostanti, che riproducono i primi quattro versi di entrambi i brani.

$\begin{array}{lllllllll}\text { I } & \text { II } & \text { III } & \text { IV } & \text { V } & \text { VI } & \text { VII } & \text { VIII } & \text { IX } \\ & & & & & & & \\ & & & \mathrm{x} & & & & \mathrm{x} & \\ & \mathrm{x} & & \mathrm{x} & & \mathrm{x} & & \mathrm{x} & \\ \mathrm{x} & \mathrm{x} & \mathrm{x} & \mathrm{x} & \mathrm{x} & \mathrm{x} & \mathrm{x} & \mathrm{x} & \mathrm{x}\end{array}$

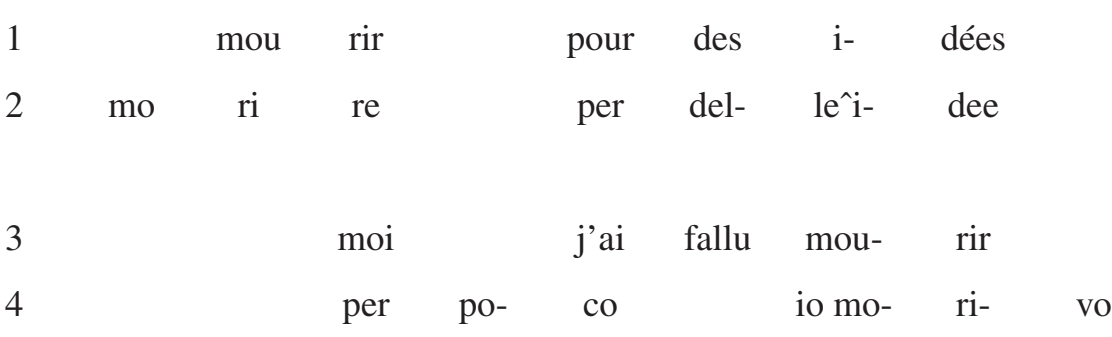

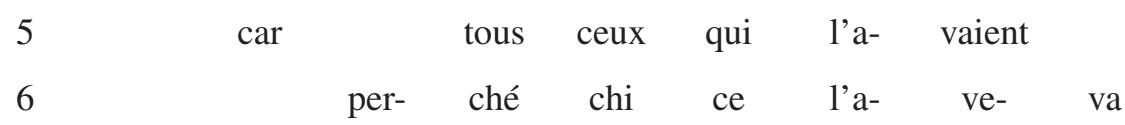
gri- dan- do vi- va la mor-

\section{$X$}


(B2)

$\begin{array}{lllllllll}\text { I } & \text { II } & \text { III } & \text { IV } & \text { V } & \text { VI } & \text { VII } & \text { VIII } & \text { IX }\end{array}$

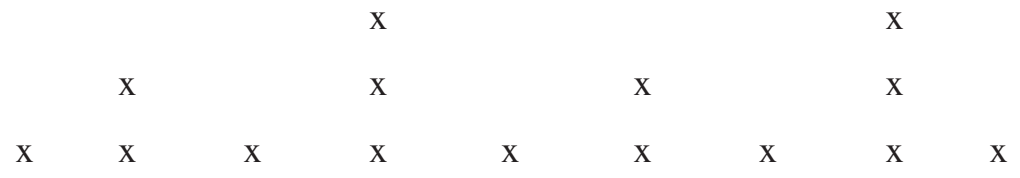

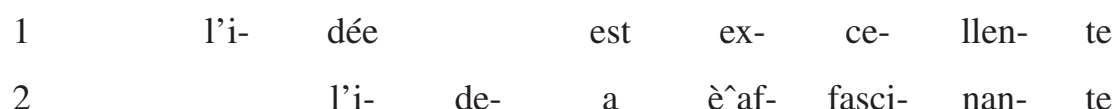

3 de ne l'a- voir pas eu

4 sen- zâa- ver- la mai^a- vu- ta

5 mul- ti- tu- de^a cca- blan- te

6 u- na fol- la di gen- te

7 me sont tom- bés de- ssus

8 pro- priôad- dos- $\quad$ so mi è ca- du- ta

Ancora una volta si osserva come l'aderenza alla lettera del testo sia molto meno vincolante dell'aderenza alla struttura metrica; nei pochi casi in cui non si riesca a conciliare lessico e ritmo ( «avec un soupçon de réserve toutefois» > «dicendomi peraltro in separata sede» - prima strofa, non inserito nella griglia), sarà sempre il primo a farne le spese.

Interessante è anche il caso del verso che funge da ritornello: la traduzione del «d'accord» originario con l'italiano «d'accordo» o avrebbe provocato mismatch (esempio $2^{*}$,III della griglia $\mathrm{B}^{3}$ ), oppure uno spostamento di accenti rispetto al testo francese (esempio $2^{* *}$ ) che avrebbe inoltre condotto a uno sgradito allungamento della struttura melodica rispetto al brano origi- 
nale. Per ovviare a tali inconvenienti De André sceglie di utilizzare di un vocabolo bisillabo ossitono («vabbè»), esattamente come nel brano originale: la difficoltà nel reperire parole di questo tipo nella lingua italiana fa propendere per una decisione cosciente e altamente voluta dal cantautore italiano.

$\begin{array}{llllllllllll}\text { (B3) } & \text { I } & \text { II } & \text { III } & \text { IV } & \text { V } & \text { VI } & \text { VII } & \text { VIII } & \text { IX } & \text { X } & \text { XI }\end{array}$

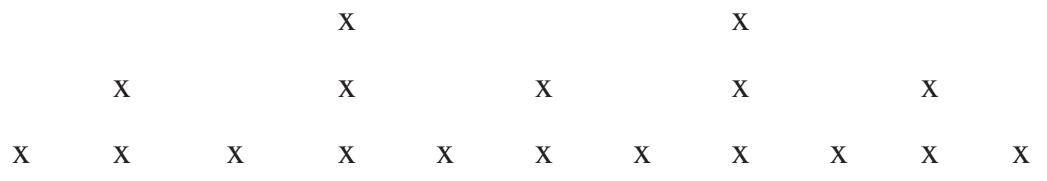

$\begin{array}{llcccccc}1 & \text { d'a- } & \text { ccord mais } & \text { de } & \text { mort } & \text { len- te } \\ 2 & \text { vab- } & \text { bè } & \text { ma } & \text { di } & \text { mor- te len- ta }\end{array}$

2* d'ac- cor- do ma di mor- te len- ta

$2^{* *} \quad$ d'ac- cor- do ma di mor- te len- ta

\subsection{Siete per sempre coinvolti}

Un caso di studio altrettanto interessante è la traduzione di De André di «Chacun de vous est concerné» (Dominique Grange). L'esempio è paradigmatico perché, oltre alla versione originale, inserita nel concept album «Storia di un impiegato», esiste una seconda versione (probabilmente più antica di quella riprodotta nell'album) che venne cantata in più occasioni dall' artista durante i suoi concerti, e che dunque era considerata non inferiore rispetto alla versione ufficiale. 


\section{Chacun de vous est concerné} (Grange)

Même si le mois de mai Ne vous a guère touché Même s'il n'y a pas eu De manif' dans votre rue Même si votre voiture n'a pas été incendiée Même si vous vous en foutez. Chacun de vous est concerné

Même si vous avez feints

De croire qu'il se passait rien Quand dans le pays entier Des usines s'arrêtaient Même si vous n'avez rien fait Pour aider ceux qui luttaient Même si vous vous en foutez. Chacun de vous est concerné

Même si vous avez fermé Votre porte à notre nez Une nuit où nous avions Les CRS aux talons Si vous nous avez laissés Matraqués sur le palier Même si vous vous en foutez.

Chacun de vous est concerné

Même si dans votre ville Tout est resté bien tranquille Sans pavés, sans barricades Sans blessés et sans grenades Même si vous avez gobé Ce que disait la télé Même si vous vous en foutez Chacun de vous est concerné

Même si vous croyez maintenant Que tout est bien comme avant Parce que vous avez voté L'ordre et la sécurité

\section{La canzone del maggio}

(De André, versione I)

Anche se il nostro maggio

Ha fatto a meno del vostro coraggio

Se la paura di guardare

Vi ha fatto chinare il mento

Se il fuoco ha risparmiato

Le vostre Millecento

Anche se voi vi credete assolti

Siete lo stesso coinvolti

E se vi siete detti

Non sta succedendo niente

Le fabbriche riapriranno

Arresteranno qualche studente

Convinti che fosse un gioco

A cui avremmo giocato poco

Provate pure a credevi assolti

Siete lo stesso coinvolti

Anche se avete chiuso

Le vostre porte sul nostro muso

La notte che le pantere

Ci mordevano il sedere

Lasciamoci in buonafede

Massacrare sui marciapiede

Anche se ora ve ne fregate

Voi quella notte voi c'eravate

E se nei vostri quartieri

Tutto è rimasto come ieri

Senza le barricate

Senza feriti senza granate

Se avete preso per buone

Le verità della televisione

Anche se allora vi siete assolti

Siete lo stesso coinvolti

E se credente ora

Che tutto sia come prima

Perché avete votato ancora

La sicurezza la disciplina

\section{La canzone del maggio}

(De André, versione II)

Anche se il nostro maggio

Ha fatto a meno del vostro coraggio Se la paura di guardare

Vi ha fatto guardare in terra

Se avete deciso in fretta

Che non era la vostra guerra

Voi non avete fermato il vento

Gli avete fatto perdere tempo

E se vi siete detti

Non sta succedendo niente

Le fabbriche riapriranno

Arresteranno qualche studente

Convinti che fosse un gioco

A cui avremmo giocato poco

Voi siete stati lo strumento

Per farci perdere un sacco di tempo

Se avete lasciato fare

Ai professionisti dei manganelli

Per liberarvi di noi canaglie

Di noi teppisti di noi ribelli

Lasciamoci in buonafede

Sanguinare sui marciapiede

Anche se ora ve ne fregate

Voi quella notte voi c'eravate

E se nei vostri quartieri

Tutto è rimasto come ieri

Se sono rimasti a posto

Perfino i sassi nei vostri viali

Se avete preso per buone

Le verità dei vostri giornali

Non vi è rimasto nessun argomento

Per farci ancora perdere tempo

Lo conosciamo bene

Il vostro finto progresso

Il vostro comandamento

Ama il consumo come te stesso 


$\begin{array}{lll}\text { Même si vous ne voulez pas } & \text { Convinti di allontanare } & \text { E se voi lo avete osservato } \\ \text { Que bientôt on remette ça } & \text { La paura di cambiare } & \text { Fino ad assolvere chi ci ha sparato } \\ \text { Même si vous vous en foutez } & \text { Verremo ancora alle vostre porte } & \text { Verremo ancora alle vostre porte } \\ \text { Chacun de vous est concerné } & \text { E grideremo ancora più forte } & \text { E grideremo ancora più forte } \\ & \text { Per quanto voi vi crediate assolti } & \text { Voi non potete fermare il vento } \\ & \text { Siete per sempre coinvolti } & \text { Gli fate solo perdere tempo } \\ & \text { Per quanto voi vi crediate assolti } & \text { Voi non potete fermare il vento } \\ & \text { Siete per sempre coinvolti } & \text { Gli fate solo perdere tempo }\end{array}$

Mentre la versione ufficiale (d'ora in avanti «versione I», vs «versione II») è assolutamente fedele alla lettera del testo francese, la versione II in certi punti se ne discosta in maniera siginificativa, come a voler far risaltare la propria autonomia rispetto al modello. Ciononostante, entrambe le versioni mantengono la medesima riverenza nei confronti dello schema ritmico-melodico dell'originale, ed entrambe ne rispettano la struttura metrica alla stessa maniera.

Il testo di Dominique Grange è composto interamente da sénaires e heptasyllabes, mentre i versi delle due versioni di De André possiedono un range di sillabe che varia dal settenario («e se credete ora», «lo conosciamo bene») fino al decasillabo/endecasillabo («anche se allora vi siete assolti», «non vi è rimasto nessun argomento»). Tuttavia, è rilevante il fatto che, come nei precedenti esempi, tanto nella prima quanto nella seconda versione non esista uno scarto significativo, da un punto di vista metrico, rispetto alla canzone originale: ennesimo indizio in favore dell'ipotesi che, nel tradurre il brano francese, De André abbia dato una netta priorità all'aspetto metricomelodico e, solo in seguito, si sia occupato della scelta di vocaboli da inserire all'interno di tale griglia.

\section{DA CANZONI (INGLESI) A CANZONI}

Con il cambiamento del supporto linguistico del testo di partenza si sarebbero dovute rilevare - o almeno queste erano le aspettative - delle modifiche significative anche a livello di modalità di traduzione. Tuttavia, la traduzione di canzoni inglesi sembra avvenire esattamente con le stesse peculiarità già notate nello studio della traduzione di canzoni francesi: il rispetto della melodia originale è il vincolo più alto in gerarchia, e da esso procedono tutti gli altri, ivi compreso il rispetto per la lettera del testo. 


\begin{tabular}{|c|c|c|c|c|c|c|c|c|c|c|c|c|c|}
\hline \multirow{2}{*}{\multicolumn{2}{|c|}{ (C) }} & II & III & IV & V & VI & VII & VIII & IX & $X$ & XI & XII & XIII \\
\hline & & & $\mathrm{x}$ & & & & $\mathrm{x}$ & & & & $\mathrm{x}$ & & \\
\hline & $\mathrm{x}$ & & $\mathrm{x}$ & & $\mathrm{X}$ & & $\mathrm{X}$ & & $\mathrm{x}$ & & $\mathrm{X}$ & & $\mathrm{X}$ \\
\hline & $\mathrm{x}$ & $\mathrm{x}$ & $\mathrm{X}$ & $\mathrm{X}$ & $\mathrm{X}$ & $\mathrm{x}$ & $\mathrm{X}$ & $\mathrm{x}$ & $\mathrm{X}$ & $\mathrm{X}$ & $\mathrm{X}$ & $\mathrm{X}$ & $\mathrm{X}$ \\
\hline
\end{tabular}

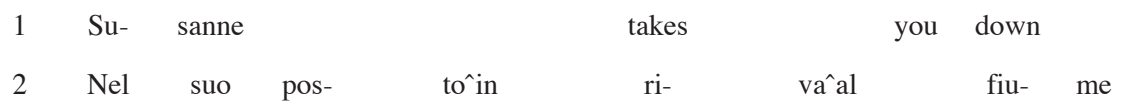

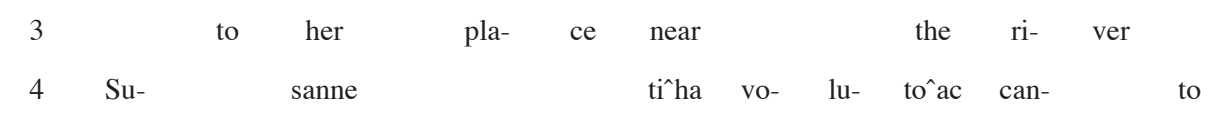

$\begin{array}{llllllll}5 & \text { you } & \text { can hear the boats } & \text { go } & \text { by } \\ 6 & \mathrm{e}^{\wedge} \text { râs- col- } & & \text { ti^an- dar } & \text { le } & \text { bar } & \text { che }\end{array}$

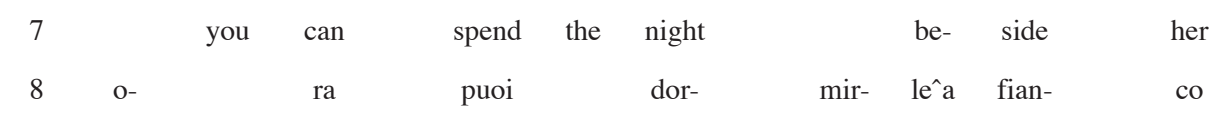

$\begin{array}{cccccccc}9 & \text { and } & \text { you } & \text { know } & \text { she’s } & \text { half } & \text { cra- } & \text { zy } \\ 10 & \text { sì } & \text { lo } & \text { sai } & & \text { che leî̀ } & \text { paz- } & \text { za }\end{array}$

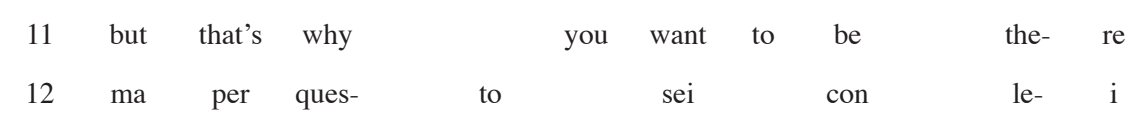

\begin{tabular}{|c|c|c|c|c|c|c|c|}
\hline 13 & and & she & feeds & you & tea & and & ran- \\
\hline 14 & $\mathrm{E}$ & $\mathrm{ti}$ & of- & fre^il & tè̀e & le^a- & ran- \\
\hline
\end{tabular}

$\begin{array}{lllllllll} & \text { come } & \text { all } & & \text { the } & \text { way } & \text { from } & \text { Chi- } & \text { na } \\ 16 & \text { che^ha } & \text { por- } & \text { ta- } & \text { to } & \text { dal- } & \text { la } & \text { Ci- } & \text { na }\end{array}$




\subsection{Suzanne}

Nel 1972 De André pubblica un singolo in cui sono presenti due soli brani, entrambi frutto di traduzioni di canzoni del cantautore canadese Leonard Cohen. Uno di essi, Suzanne, è di seguito analizzato.

Così come per la traduzione delle canzoni francesi, anche per quelle inglesi De André si attiene a uno scrupoloso rispetto per la melodia - e dunque per la griglia metrica - del brano di partenza; la fedeltà lessicale al testo originale è di conseguenza rispettata solo lì dove questo primo criterio è pienamente soddisfatto.

\subsection{Famosa volpe azzurra}

La canzone di Cohen «Famous Blue Raincoat» (1971) fu tradotta da De André per Ornella Vanoni, la quale la inserì nel suo album «Ricetta di donna» del 1980. La traduzione di questa canzone dovette impegnare non poco il cantautore perché, rispetto alla scena originaria, viene invertito il sesso dei protagonisti: non è più la lettera di un uomo a un altro uomo, bensì quella di una donna a una donna (con tutti i trasferimenti di genere che ne derivano). Parimenti, l'ambientazione è spostata da New York a Milano.

Come già per i brani francesi, l'unica condizione a cui De André è interessato (molto più della conservazione del numero di sillabe dei versi inglesi) è il rispetto della griglia metrica originaria, ottenuto a volte anche tramite espedienti non canonici (ed esteticamente discutibili) come l'aferesi di QUESTE > 'STE (strofa 8; cfr. griglia D). 
Famous Blue Raincoat (Cohen)

It's four in the morning

The end of December

I'm writing you now just to see

If you're better

New York is cold but I

Like where I'm living there's

Music on Clinton Street all

Through the evening

I hear that you're building

Your little house

Deep in the desert

You're living for nothing now

I hope you're keeping

Some kind of record

Yes, and Jane

Came

By with a lock of your hair

She said that you gave it to her

That night that you planned to go cle-

ar

Did you ever go clear

Ah the last time we saw you

You looked so much older

Your famous blue raincoat

Was torn at the shoulder

You'd been to the station

To meet every train and

You came home

Without Lili Marleen

And you treated

My woman

To a flake

Of your life

And when she came back

She was nobody's wife

Well I see
Famosa volpe azzurra (trad. De André)

Le quattro di sera

Di fine dicembre

Ti scrivo e non so

Se ci servirà a niente

Milano è un po' fredda

Ma qui vivo bene

Si fa musica all' "Angolo"

Quasi tutte le sere

Mi dicono stai arredando

La tua piccola casa

In qualche deserto

E che per il momento

Stai vivendo di poco

O soltanto di quello

Sì

E Lucio sai

Parla ogni tanto di te

Di quella notte in cui tu

Gli hai detto che eri sincera

Sei mai stata sincera

L'ultima volta

Ti ho vista invecchiata

Con la tua volpe azzurra

Famosa e sciupata

Lì alla stazione

A contare mille treni

E tornartene a casa

Come Lili Marlene

Hai trattato il mio uomo

Come un fiocco di neve

Che si scioglie da sé

$E$ un attimo dopo

Non era più l'uomo

Né per te né per me

E ti vedo lì 
You

There with the rose in your teeth One more thin gypsy thief

Well I see Jane's awake

She sends her regards

And what can I tell you

My brother my killer

What can I possibly say

I guess that I miss you

I guess I forgive you

I'm glad you stood in my way

If you ever come by here

For Jane or for me

Your enemy is sleeping

And his woman is free

Yes

And thanks

Tor the trouble you took

From her eyes

I thought it was there

For good

So I never tried

And Jane

Came

By with a lock of your hair

She said that you gave it to her

That night that you planned to go clear

Sincerely

L. Cohen
Con

Una rosa tra i denti

Un trucco nuovo per nuovi clienti

Ora Lucio si é svegliato

Anche lui ti saluta

Che cosa altro dirti

Sorella assassina

Che cosa altro scriverti

Adesso non so

Se non che mi manchi

Se non che ci manchi

E certo alla fine

Ti perdonerò

E se tornerai da 'ste parti

Per lui o per noi

Troverai una rivale che dorme

E il suo uomo se vиoi

E grazie

Per la noia che gli hai tolto

Dagli occhi

Io mi c'ero abituata e così

Non mi ero neppure provata

E Lucio

Sai

Parla ogni tanto di te

Di quella volta che tu

Gli hai fatto la notte più bella

Ti saluto

Tua Ornella 
Come già per i brani francesi, l'unica condizione a cui De André è interessato (molto più della conservazione del numero di sillabe dei versi inglesi) è il rispetto della griglia metrica originaria, ottenuto a volte anche tramite espedienti non canonici (ed esteticamente discutibili) come l'aferesi di QUESTE > 'STE (strofa 8; cfr. griglia D).

$\begin{array}{llllllllllllll}\text { (D) } & \text { I } & \text { II } & \text { III } & \text { IV } & \text { V } & \text { VI } & \text { VII } & \text { VIII } & \text { IX } & \text { X } & \text { XI } & \text { XII } & \text { XIII }\end{array}$

\begin{tabular}{|c|c|c|c|c|c|c|c|c|c|c|c|}
\hline & & & $\mathrm{x}$ & & & & $\mathrm{x}$ & & & & $\mathrm{x}$ \\
\hline & $\mathrm{X}$ & & $\mathrm{X}$ & & $\mathrm{X}$ & & $\mathrm{x}$ & & $\mathrm{X}$ & & $\mathrm{X}$ \\
\hline X & $\mathrm{x}$ & $\mathrm{x}$ & $\mathrm{X}$ & $\mathrm{x}$ & $\mathrm{X}$ & $\mathrm{x}$ & $\mathrm{x}$ & $\mathrm{x}$ & $\mathrm{X}$ & $\mathrm{x}$ & $\mathrm{X}$ \\
\hline & if & you & e- & ver & come & & & & by & & here \\
\hline ( & se & & tor- & ne- & rai & & & & $\mathrm{da}$ & ste & par- \\
\hline
\end{tabular}

Il brano di Cohen è melodicamente tripartito. Un identico motivo musicale unisce le strofe 1, 4 e 7; un altro le strofe 2, 5 e 8; un ultimo motivo accomuna le strofe 3, 6 e 9. La stessa successione, ancora una volta, si ritrova nella traduzione di De André, che mantiene la struttura melodica dell'originale nonostante la sua complessità.

La melodia attrae lo schema metrico. Di conseguenza, sia in Cohen che in De André le strofe melodicamente affini sono composte da un numero di sillabe simile fra loro, sia in verticale - da strofa a strofa - che in orizzontale, da testo inglese a italiano (ad esempio: le strofe 1, 4 e 7 del brano di Cohen sono composte da versi della stessa lunghezza, i quali a loro volta sono della stessa lunghezza dei versi delle strofe 1, 4 e 7 del brano di De André).

A tale regola generale fa eccezione la strofa 5 . I primi quattro versi di Cohen sono estremamente più corti rispetto ai corrispettivi delle strofe 2 e 8 (ma la melodia di questa stanza rimane immutata rispetto a quella delle sue strofe «sorelle»): per la precisione, la lunghezza dei versi è, solo in questo caso, 3' 2' 2' 3' 5 6'. De André, di contro, tradisce l'aderenza alla metrica della strofa inglese in ossequio alla fedeltà allo schema metrico complessivo; difatti egli conserva anche in questo caso la «corretta» lunghezza metrico-si- 
llabica («corretta», ossia identica a quella delle strofe 2 e 8 ), producendo una strofa composta da versi con schema: 6' 6' 6 5' 5' 6.

Una volta di più si può dunque osservare il medesimo fenomeno: nella traduzione di canzoni, l'aderenza allo schema metrico-melodico preso come modello è, in De André, più forte di qualsiasi altro vincolo, compresa l'aderenza alla lettera del testo o perfino alle deroghe metriche contenute in uno specifico punto (come nell'esempio della strofa 5) dello stesso testo di partenza.

Di contro, nell'adattamento in musica di brani poetici l'assenza di una melodia originaria comporterà notevoli stravolgimenti nel modo di tradurre adottato dal cantautore italiano.

\section{DA POESIA A MUSICA}

Un intero album dell'artista genovese («Non al denaro non all'amore né al cielo», 1971) fu consacrato alla trasposizione musicale di una selezione di componimenti tratti dall'antologia di Spoon River di Edgar Lee Masters.

La libertà metrica dei versi di Masters - resa possibile dal suo essere svincolata da qualsiasi tipo di schema melodico - non poteva essere duplicata in brani destinati invece ad essere musicati, e che di conseguenza dovevano necessariamente fondarsi su un pattern metrico relativamente regolare.

Non avendo dunque in questo contesto (contrariamente ai casi precedentemente analizzati) un modello metrico-melodico da dover seguire, De André si trovò nella situazione di doverne creare uno ex-novo per ogni singola canzone, sentendosi così al contempo ancora meno vincolato alla lettera della propria fonte che non in precedenza (dove, pur con i limiti osservati, sempre cercò di rispettare almeno la struttura argomentativa e il senso generale di ogni singola strofa tradotta). È infatti solo in questo album che il testo di base venne per la prima volta utilizzato più come un modello da cui prendere spunto per creare brani autonomi che non - come invece era avvenuto nelle traduzioni di canzoni - come un testo da tradurre con il maggior grado possibile di fedeltà. 


\subsection{La collina}

Così come la poesia «The Hill» apre l'antologia di Spoon River, allo stesso modo «La collina» è la canzone introduttiva dell'album «Non al denaro non all'amore né al cielo».

In questa prima trasposizione in musica si nota subito un carattere che sarà ancora più accentuato nei brani successivi, ossia la (quasi) totale autonomia del testo italiano rispetto al suo modello originale ${ }^{5}$.

\section{The Hill (Masters)}

Where are Elmer, Herman, Bert, Tom and Charley,

The weak of will, the strong of arm, the clown, the boozer, the fighter? All, all, are sleeping on the hill.

One passed in a fewer,

One was burned in a mine,

One was killed in a brawl,

One died in a jail,

One fell from a bridge toiling for children and wife-

All, all are sleeping, sleeping, sleeping on the hill.

Where are Ella, Kate, Mag, Lizzie and Edith,

The tender heart, the simple soul, the loud, the proud, the happy one?

All, all, are sleeping on the hill.

One died in shameful child-birth,

One of a thwarted love,

One at the hands of a brute in a brothel,

One of a broken pride, in the search for heart's desire,

One after life in far-away London and Paris

Was brought to her little space by Ella and Kate and Mag-

All, all are sleeping, sleeping, sleeping on the hill.

Where are Uncle Isaac and Aunt Emily,

And old Towny Kincaid and Sevigne Houghton,

And Major Walker who had talked

With venerable men of the revolution? -

\section{La collina (De André)}

Dove se n'è andato Elmer

Che di febbre si lasciò morire

Dov'è Herman bruciato in miniera

Dove sono Bert e Tom

Il primo ucciso in una rissa

E l'altro che uscì già morto di galera

E cosa ne sarà di Charley

Che cadde mentre lavorava

Dal ponte volò volò sulla strada

Dormono dormono sulla collina

Dormono dormono sulla collina

Dove sono Ella e Kate

Morte entrambe per errore

Una di aborto l'altra d'amore

E Maggie uccisa in un bordello

Dalle carezze di un animale

E Edith consumata da uno strano

male

E Lizzie che inseguì la vita

Lontano e dall'Inghilterra

$F u$ riportata in questo palmo di terra

Dormono dormono sulla collina

Dormono dormono sulla collina

\footnotetext{
${ }^{5}$ I testi inglesi sono tratti da Masters $2001^{10^{*}}$.
} 
All, all, are sleeping on the hill.

They brought them dead sons from the war,

And daughters whom life had crushed,

And their children fatherless, crying -

All, all are sleeping, sleeping, sleeping on the hill.

Where is Old Fiddler Jones

Who played with life all his ninety years,

Braving the sleet with bared breast,

Drinking, rioting, thinking neither of wife nor kin, Nor gold, nor love, nor heaven?

Lo! he babbles of the fish-frys of long ago,

Of the horse-races of long ago at Clary's Grove,

Of what Abe Lincoln said

One time at Springfield.

\author{
Dove sono i generali \\ Che si fregiarono nelle battaglie \\ Con cimiteri di croci sul petto \\ Dove i figli della guerra \\ Partiti per un ideale \\ Per una truffa per un amore finito male \\ Hanno rimandato a casa \\ Le loro spoglie nelle bandiere \\ Legate strette perché sembrassero intere \\ Dormono dormono sulla collina \\ Dormono dormono sulla collina \\ Dov'è Jones il suonatore \\ Che fu sorpreso dai suoi novant'anni \\ E con la vita avrebbe ancora giocato \\ Lui che offrì la faccia al vento \\ La gola al vino e mai un pensiero \\ Non al denaro non all'amore né al cielo \\ Lui sì sembra di sentirlo \\ Cianciare ancora delle porcate \\ Mangiate in strada nelle ore sbagliate \\ Sembra di sentirlo ancora \\ Dire al mercante di liquore \\ "Tu che lo vendi cosa ti compri di migliore?"
}

I versi di De André, pur oscillando fra lunghezze di valori apparentemente molto diversi fra loro (fra le sette e le quindici sillabe), mantengono comunque una loro unità ritmica, con accenti interni abbastanza regolari.

Rispetto alla poesia originale, inoltre, la canzone fa un uso più abbondante - ma pur sempre non organico - di rime e assonanze, perlopiù in rima biaciata (miniera : galera; errore : amore; animale : male; generali : ideale : male; pensiero : cielo; porcate : sbagliate). Si registra una costante: quella di chiudere ogni strofa con un distico di versi in rima (lavorava: strada; Inghilterra : terra; bandiere : intere; liquore : migliore).

Il testo di partenza è spesso stravolto: sistematica, ad esempio, è la compressione di due blocchi poetici in uno durante le enumerazioni dei personaggi (mentre, nella poesia, la prima strofa di ogni blocco semantico è dedicata alla lista dei nomi dei protagonisti - «Where are Elmer, Herman, 
Bert, Tom and Charley»- e la successiva alla loro rapida descrizione «One passed in a fewer...»-, nella canzone i due passaggi vengono condensati in uno: «Dove se n'è andato Elmer I che di febbre si lasciò morire»). Parimenti, sono facilmente rinvenibili nel brano di De André degli elementi innovativi rispetto al testo americano. Si vedano ad esempio i versi che chiudono la poesia («Of what Abe Lincoln said I One time at Springfield»), e che si riferiscono a una frase scritta da Abraham Lincoln a suo fratello il 4 novembre 1851 (estratto da Nicolay, 2004: 48):

When I came into Charleston, day before yesterday, I learned that you were anxious to sell the land where you live and move to Missouri. I have been thinking of this ever since, and cannot but think such a notion is utterly foolish. What can you do in Missouri better than here?

Nella poesia di Masters, dunque, la frase che Jones farfuglierebbe si riferisce al fatto che non ha senso trasferirsi in un luogo peggiore di quello in cui già si vive (ma è solo con un notevole sforzo ermeneutico che il lettore può intendere il significato di tali versi). Nel brano di De André la stessa affermazione viene doppiamente stravolta: da un lato, ciò che era implicito («Of what Abe Lincoln said»: but what did he say?, si chiede il lettore) diviene esplicito; dall'altro, l'oggetto della riflessione è radicalmente modificato - dal trasloco in un altro stato a un discorso sul vino (che rimanda alla leggerezza con cui Jones affronta la vita) - divenendo tuttavia perfettamente appropriato al personaggio qui descritto: «sembra di sentirlo ancora I dire al mercante di liquore I tu che lo vendi cosa ti compri di migliore?».

\subsection{Il suonatore Jones}

Il personaggio del suonatore Jones compare - evento unico - in due canzoni diverse: e, dopo che la sua comparsa aveva segnato la fine del primo brano, la sua nuova apparizione segna la fine dell'intero album.

Il fenomeno notato sopra si accentua in questo testo: De André, quando si trova a tradurre poesie (e non canzoni), utilizza la sua fonte con estrema libertà. Rispetto a «La collina», il caso presente è ancora più eclatante per due motivi: da un lato, la regolare struttura metrica della poesia avrebbe permesso, in linea teorica, una sua precisa trasposizione in versi italiani; dall'altro, proprio rispetto a «La collina» la fedeltà - anche semantica - al testo originale è, di contro, ancora meno marcata, anzi, si può dire che De André 


\section{prese alcune frasi della poesia americana come impalcatura generale della} sua canzone, per poi costruire a partire da esse un brano del tutto autonomo.

\section{Fiddler Jones (Masters)}

The earth keeps some vibration going There in your heart, and that is you. And if the people find you can fiddle, Why, fiddle you must, for all your life. What do you see, a harvest of clover? Or a meadow to walk through to the river?

The wind's in the corn; you rub your hands For beeves hereafter ready for market; Or else you hear the rustle of skirts Like the girls when dancing at Little Grove. To Cooney Potter a pillar of dust Or whirling leaves meant ruinous drouth; They looked to me like Red-Head Sammy Stepping it off, to Toor-a-Loor.

How could I till my forty acres Not to speak of getting more,

With a medley of horns, bassoons and piccolos Stirred in my brain by crows and robins And the creak of a wind-mill - only these? And I never started to plow in my life That some one did not stop in the road And take me away to a dance or picnic. I ended up with forty acres; I ended up with a broken fiddleAnd a broken laugh, and a thousand memories, And not a single regret.

\section{Il suonatore Jones (De André)}

In un vortice di polvere

Gli altri vedevan siccità

A me ricordava

La gonna di Jenny

In un ballo di tanti anni fa

Sentivo la mia terra

Vibrare di suoni era il mio cuore

E allora perché coltivarla ancora

Come pensarla migliore

Libertà l'ho vista

Dormire nei campi coltivati

A cielo e denaro

A cielo ed amore

Protetta da un filo spinato

Libertà l'ho vista

Svegliarsi ogni volta che ho suonato Per un fruscio di ragazze a un ballo

Per un compagno ubriaco

E poi se la gente sa

E la gente lo sa che sai suonare

Suonare ti tocca

Per tutta la vita

E ti piace lasciarti ascoltare

Finii con i campi alle ortiche

Finii con un flauto spezzato

$E$ un ridere rauco e ricordi tanti

E nemmeno un rimpianto 
Lo schema metrico del testo di De André è, rispetto agli schemi della canzone d'autore italiana, relativamente articolato (e relativamente stabile da strofa a strofa), e sicuramente molto più complesso di quello della poesia da cui la canzone trae spunto. Di seguito una tabella con il numero di sillabe (contate fino all'ultimo accento tonico) delle tre strofe:

$\begin{array}{ccc}\text { I } & \text { II } & \text { III } \\ 7 & 7 & 7 \\ 8 & 8 & 10 \\ 5 & 5 & 5 \\ 5 & 5 & 5 \\ 9 & 9 & 9 \\ 6 & 6 & 8 \\ 10 & 10 & 8 \\ 10 & 10 & 10 \\ 7 & 7 & 6\end{array}$

Come anticipato, la fedeltà alla poesia è rispettata solo a tratti. Si riconoscono, nella canzone, alcuni versi del testo americano: «And if the people find you can fiddle, I Why, fiddle you must, for all your life» $(=\langle\mathrm{E}$ poi se la gente sa I e la gente lo sa che sai suonare I suonare ti tocca I per tutta la vita»); «Or else you hear the rustle of skirts. I Like the girls when dancing at Little Grove» (= «per un fruscio di ragazze a un ballo»); «I ended up with forty acres; I I ended up with a broken fiddle - I And a broken laugh, and a thousand memories, I And not a single regret» (= «finii con $\mathrm{i}$ campi alle ortiche I finii con un flauto spezzato I e un ridere rauco e ricordi tanti le nemmeno un rimpianto»; si noti, fra l'altro, che il riddle del testo originale diventa, forse per esigenze metriche, un flauto).

A parte questi pochi spunti - necessari per giustificare lo statuto stesso di «traduzione»-, De André reinterpretò in maniera autonoma testo e melodia. Si possono avanzare due ipotesi: o il cantautore decise di modificare prima il testo, e solo in seguito la struttura metrica, o viceversa. Sulla base di quanto finora osservato, sembra plausibile concludere che fu proprio la necessità di costruire una nuova griglia metrico-melodica che fece sentire l'artista geno- 
vese al tempo stesso meno vincolato anche al rispetto della lettera del testo originale.

\subsection{Un malato di cuore}

Anche questo terzo (e ultimo) esempio mostra come l'approccio di De André alla traduzione dell'antologia di Spoon River sia stato totalmente differente rispetto a quello mostrato nella traduzione delle canzoni di Cohen e Brassens.

La strofa di Masters traccia, in pochissimi versi, il ritratto di un giovane morto a causa di problemi cardiaci. Il componimento di De André amplia in maniera significativa il testo americano aggiungendo interi brani a partire da spunti derivanti dalla poesia, i cui versi iniziali permangono tuttavia facilmente rintracciabili ( $«$ In manhood I could only sip the cup, | Not drink» $=\ll e$ mai poter bere alla coppa d'un fiato I ma a piccoli sorsi interrotti»; «Kissing her with my soul upon my lips I It suddently took flight»= «e l'anima d'improvviso prese il volo»).

Entrambi i primi due distici di «Francis Turner» danno vita a due strofe che, pur essendo più articolate, sviluppano i medesimi temi dell'originale:

\section{Francis Turner (Masters)}

I could not run or play

in boyhood.

In manhood I could only sip the cup,

Not drink -

For scarlet-fever left my heart diseased.

Yet I lie here

Soothed by a secret none but Mary

knows:

There is a garden of acacia,

Catalpa trees, and arbors sweet with vines -

There on that afternoon in June

By Mary's side-

Kissing her with my soul upon my lips

\section{Un malato di cuore (De André)}

Cominciai a sognare anch'io insieme a loro

Poi l'anima d'improvviso prese il volo

Da ragazzo spiare $i$ ragazzi giocare Al ritmo balordo del tuo cuore malato E ti viene la voglia di uscire e provare Che cosa ti manca per correre al prato E ti tieni la voglia e rimani a pensare Come diavolo fanno a riprendere fiato

Da uomo avvertire il tempo sprecato A farti narrare la vita dagli occhi E mai poter bere alla coppa d'un fiato ma 
It suddently took flight.

A piccoli sorsi interrotti

E mai poter bere alla coppa d'un fiato ma

A piccoli sorsi interrotti

Eppure un sorriso io l'ho regalato E ancora ritorna in ogni sua estate Quando io la guidai o fui forse guidato

A contarle i capelli con le mani sudate

Non credo che chiesi promesse al suo sguardo

Non mi sembra che scelsi il silenzio o la voce

Quando il cuore stordì e ora no non ricordo

Se fu troppo sgomento o troppo felice

E il cuore impazzì e ora no non ricordo

Da quale orizzonte sfumasse la luce

E fra lo spettacolo dolce dell'erba Fra lunghe carezze finite sul volto Quelle sue cosce color madreperla Rimasero forse un fiore non colto Ma che la baciai questo sì lo ricordo Col cuore ormai sulle labbra Ma che la baciai per Dio sì lo ricordo E il mio cuore le restò sulle labbra

E l'anima d'improvviso prese il volo Ma non mi sento di sognare con loro No non mi riesce di sognare con loro

l'impossibilità del protagonista di correre insieme ai suoi coetanei e la metafora del non «poter bere alla coppa d'un fiato».

Anche la seconda parte della poesia, incentrata sull'amore per una ragazza che Francis riuscì solo a baciare, è - nella canzone italiana - notevolmente ampliata, e occupa l'ampiezza complessiva di tre strofe. 
Ancora una volta è interessante notare l'innovazione, a livello metrico, che il cantautore attuò sul testo di partenza. Tranne poche eccezioni, i versi di De André sono tutti della medesima lunghezza, fra le undici e le dodici sillabe (con una cesura al mezzo debole ma abbastanza regolare). Le strofe, pur non essendo separate l'una dall'altra da forti pause melodiche, possono comunque essere ricondotte allo schema sopra adottato.

Il divario con il testo di partenza è palese. La volontà mimetica evidenziata nelle precedenti traduzioni delle canzoni (e già notevolmente affievolita nella trasposizione delle precedenti poesie) è qui del tutto assente, e il cantautore costruisce una struttura metrica totalmente svincolata da quella del brano originale; su questa griglia, infine, inserisce la sua personalissima «traduzione», che diviene sempre meno una traduzione e sempre più una libera reinterpretazione del brano poetico di partenza a partire dall'appropriazione e dalla rielaborazione di pochi versi sparsi.

\section{CONCLUSIONI}

Con questo articolo si è voluto indagare una piccola porzione di un problema molto più vasto: quali sono gli accorgimenti metrici adottati nella traduzione di una canzone da un sistema linguistico a un altro? Cosa cambia, a livello di modalità di traduzione, quando cambia il sistema linguistico? E, infine, quali sono le differenze nel tradurre una canzone e nel tradurre una poesia?

La metrica è coinvolta inevitabilmente in entrambe le tipologie letterarie, poesia e verso cantato. Il secondo, essendo musicato, per statuto non può prescindere dal possedere un qualche tipo di regolarità metrico-melodica. Inizialmente ci si è chiesto se le modalità di resa metrica fossero differenti a seconda della lingua di partenza, e per verificare tale affermazione si sono analizzate le traduzioni del cantautore italiano Fabrizio De André di alcune canzoni francesi e di altre inglesi. Il passo successivo è stato studiare quale gradiente di differenza ci fosse fra la traduzione di una canzone e la traduzione di una poesia, e nel farlo si è preso in considerazione il concept album «Non al denaro non all'amore né al cielo», liberamente ispirato all'antologia di Spoon River di Edgar Lee Masters.

I risultati ottenuti sono fra loro omogenei. Almeno per ciò che riguarda la produzione artistica di De André 6 , il supporto linguistico del brano origina-

${ }^{6}$ Ripetiamo ancora una volta che l'analisi del corpus lirico di un singolo cantautore era necessaria per manterenere 1'omogeneità del campione. 
le sembra essere, per gli aspetti metrici, del tutto ininfluente. Difatti, non si è verificata nessuna divergenza compositiva fra le modalità di traduzione delle canzoni di Brassens e di quelle di Cohen: la griglia metrica e la melodia del testo di partenza sono sempre state scrupolosamente rispettate. Di contro, quando la traduzione riguarda un brano poetico, che dunque non possiede una propria melodia, anche l'aderenza allo schema metrico del testo originale viene meno. Sembra dunque che il vero fattore che indirizzò, in tal senso, le scelte del cantautore non fu tanto la volontà di mantenersi fedele alla struttura metrica del brano che si accingeva a tradurre, quanto piuttosto il rispetto assoluto - qualora presenti - dei suoi aspetti musicali (da cui, ma come semplice effetto collaterale, derivò anche una maggiore aderenza alla metrica delle canzoni che non alla metrica delle poesie).

Quando una canzone traduce un'altra canzone, il traduttore non può permettersi di deviare dalla melodia di partenza; quando invece traduce una poesia, il legame con la metrica - paradossalmente - si assottiglia sempre più, fino in certi casi a scomparire.

\section{BIBLIOGRAFÍA CITADA}

DELL, F. (1989). «Concordances rythmiques entre la musique et les paroles dans le chant. L'accent et l'e muet dans la chanson française». In Le souci des apparences, M. Dominicy (ed.), 121-136. Brussels: Editions de l'Université Libre de Bruxelles.

HALLE, J. \& LERDAHL, F. (1993). «A Generative Textsetting Model». Current Musicology 55, 3-23.

HAYES, B. \& KAUN, A. (1996). «The Role of Phonological Phrasing in Chanted and Sung Verse». The Linguistic Review 13, 243-303.

HAYES, B. \& MCEACHERN, M. (1998). «Quatrain form in English folk verse». Language 74, 473-507.

LERDAHL, F. \& JACKENDOFF, R. (1983). A Generative Theory of Tonal Music. Cambridge, MA: MIT Press.

LIBERMAN, M. (1975). The Intonational System of English. Unpublished Ph.D. dissertation. Cambridge, MA: MIT Press.

MASTERS, E. L. (2001). Antologia di Spoon River. F. Pivano (ed.), Decima edizione italiana. Torino: Einaudi. 
NICOLAY, J. G. \& HAY, J. (2004). Abraham Lincoln: A History, 10 vol. 1. Ristampato Whitefish (Montana): Kessinger Publishing.

VALENTI, G. (2011). «Il ruolo del 'magico numero sette' nella canzone d'autore italiana». Cognitive Philology 4 (http://ojs.uniromal.it/index.php/cogphil/article/view/9351/9233).

Recibido el 2 de abril de 2012.

Aceptado el 17 de septiembre de 2012. 